\title{
Globe
}

Revue internationale d'études québécoises

\section{Gilles Paquet : Oublier la Révolution tranquille. Pour une nouvelle socialité, Montréal, Liber, $161 \mathrm{p}$.}

\section{Luc Turgeon}

Volume 2, numéro 2, 1999

URI : https://id.erudit.org/iderudit/1000474ar

DOI : https://doi.org/10.7202/1000474ar

Aller au sommaire du numéro

Éditeur(s)

Globe, Revue internationale d'études québécoises

ISSN

1481-5869 (imprimé)

1923-8231 (numérique)

Découvrir la revue

Citer ce compte rendu

Turgeon, L. (1999). Compte rendu de [Gilles Paquet : Oublier la Révolution tranquille. Pour une nouvelle socialité, Montréal, Liber, 161 p.] Globe, 2(2),

161-164. https://doi.org/10.7202/1000474ar d'utilisation que vous pouvez consulter en ligne.

https://apropos.erudit.org/fr/usagers/politique-dutilisation/ 


\title{
Recensions
}

\author{
Gilles Paquet \\ Oublier la Révolution tranquille. \\ Pour une nouvelle socialité \\ Montréal, Liber, 161p.
}

Gilles Paquet nous propose avec Oublier la Révolution tranquille un essai impressionniste qui, à défaut de convaincre, suscite des interrogations pertinentes en mesure de stimuler ceux qui, historiens, sociologues ou économistes, tentent à la fois de relire l'impact de la Révolution tranquille et de penser le Québec de demain. La thèse mise de l'avant par l'économiste est simple : la piètre performance de l'économie québécoise depuis les années 60 serait la conséquence de la décapitalisation sociale, fruit de «manœuvres d'invasions par l'État de la vie des Québécois» (p. 7). Cependant, l'ouvrage de Gilles Paquet est vaste et, loin de se limiter à la question du développement économique du Québec, touche un ensemble de sujets, du caractère postmoderne de la société québécoise à la nécessité d'une revalorisation de la société civile.

Après un survol des grandes étapes de la socio-économie québécoise et une critique de l'historiographie qui a cloué au pilori le régime duplessiste, l'auteur s'attarde à mettre en place un cadre conceptuel permettant de penser le Québec à partir de la transformation dans le temps de la socialité, définie comme le ciment social qui unit les individus en ensembles stables et fonctionnels (p. 57). C'est ce concept de socialité qui est au cœur de l'analyse que fait l'économiste de l'impact de la Révolution tranquille et des défis de la nouvelle économie.

S'inspirant des théories du développement des économistes néoinstitutionnalistes comme Douglas North et plus particulièrement des travaux contestés du politologue américain Robert D. Putnam,

*Recensions*, Globe. Revue intemationale d'études québécoises, vol. 2, no 2, 1999. 


\section{REVUE INTERNATIONALE D'ÉTUDES QUÉBECCOISES}

l'auteur considère que la croissance économique est le fruit d'un riche soubassement socioculturel, mesuré en terme de capital social, qui facilite «l'interaction des personnes et des autres acteurs socioéconomiques et donc la création de valeurs ajoutée» (p. 87). Cette théorie fort contestée - contestations que l'auteur admet sans pour autant tenter d'en défendre les principales prémisses - permet à l'auteur d'affirmer que le bagage institutionnel et culturel traditionnel de la période précédent la Révolution tranquille (construit autour des pôles que sont la famille, la communauté et la religion) aurait contribué à la forte croissance économique sous Duplessis. La Révolution tranquille aurait à l'opposé, par une "orgie de centralisation administrative», décapité ce capital social et mené à l'échec des tentatives de concertation à la Québec inc. et au déclin du mouvement coopératif. Selon l'auteur, "moins on convivialise, moins on se parle, moins on interagit, moins on est amené à parler d'affaires et à coopérer» (p. 100).

Gilles Paquet nous propose donc dans ce livre une nouvelle façon d'étudier le Québec qui nous semble porteuse d'une réinterprétation de l'histoire contemporaine. Le concept de socialité en particulier pourrait être un moyen d'étudier les transformations du Québec depuis l'après-guerre. Après des années d'études sur l'État québécois, le livre de Paquet nous permet d'anticiper de nouvelles recherches sur la transformation de la société civile québécoise.

La thèse principale de Gilles Paquet repose cependant sur trop peu d'observations et sur un cadre théorique trop contestable pour être convaincante. Premièrement, outre une allusion rapide aux trop peu fiables et fortement critiqués World Surveys qui mesurent l'engagement associatif dans différents pays, l'auteur n'appuie sa thèse de la décapitalisation sociale sur aucune donnée concrète. Le lecteur se demande en quoi, par exemple, le déclin du capital social aurait été plus désastreux au Québec qu'en Ontario ou qu'aux États-Unis. Si le capital social peut être un outil de développement économique, principalement dans la nouvelle économie, il ne saurait être le principal facteur. Il est difficile de croire que la croissance économique actuelle des États-Unis, par exemple, serait due à une augmentation du capital social. Notons d'ailleurs que Putnam n'a 


\section{RECENSIONS}

cessé lors des dernières années de dénoncer le déclin du capital social dans son pays.

Deuxièmement, si l'auteur affirme dans un premier temps que le développement économique dans la première moitié du siècle fut fortement influencé par des facteur exogènes, il ne mentionne pas les facteurs exogènes qui auraient pu ralentir la croissance économique du Québec dans les années subséquentes. Il fait fi de la périphérisation économique de l'économie québécoise sur le continent américain. Il occulte également la faible diversité de son économie au début des années 70 . Cette trop grande concentration dans certains secteurs industriels en déclin, en partie le fruit des politiques économiques duplessistes, serait selon certaines études récentes la principale source du déclin économique de Montréal.

Finalement, l'auteur présente une vision relativement manichéenne des relations État-société. Les interventions de l'État se font nécessairement contre la société civile et participe nécessairement à la décapitalisation sociale. Il semble oublier par le fait même que l'intervention de l'État dans plusieurs secteurs comme l'éducation, la santé et même l'économie était revendiquée au cours des années 50 par de larges secteurs de la société civile qui y voyaient une condition nécessaire à leur développement et à leur épanouissement. De plus, l'intervention active de l'État est essentielle au corporatisme ou à la concertation qu'il souhaiterait voir instaurer au Québec. Le riche soubassement culturel au Japon et en Allemagne, qu'il cite comme modèle, a été alimenté par une intervention active de l'État dans la structuration de la société civile et dans l'organisation de la solidarité.

Si on peut sans se tromper affirmer que l'intervention de l'État a contribué dans une certaine mesure à la technocratisation des rapports sociaux, il n'en demeure pas moins que la solidarité et la socialité se sont déplacées vers d'autres endroits que la famille, l'école et la communauté. Les mouvements sociaux, les lieux de travail et les groupes communautaires sont devenus de nouveaux lieux de production de la socialité qui perdurent et participent à un nouveau capital social que l'auteur occulte. Le problème, que soulève avec 


\section{REVUE INTERNATIONALE D'ÉTUDES QUÉBÉCOISES}

raison Gilles Paquet, est que l'État québécois fait peu de cas dans son mode de gestion politique de la société civile québécoise qui existe et n'a pas été totalement décapitée par la Révolution tranquille. Cependant, il s'agit d'un problème de gestion des relations Étatsociété, plutôt que de croissance économique, qui est du ressort du politique et sur lequel l'auteur aurait eu intérêt à concentrer sa critique.

Luc Turgeon Université McGill

\section{Les Langues du roman. \\ Du plurilinguisme comme stratégie textuelle}

Sous la direction de Lise Gauvin,

Les Presses de l'Université de Montréal,

«Espace littéraire», 1999, 178p.

Le présent ouvrage s'inscrit dans un courant de la critique et de la théorie littéraires qui est déjà bien établi, à savoir celui qui étudie les rapports entre la littérature et une société donnée dans l'optique de la langue littéraire comme manifestation d'une certaine hétérogénéité linguistique. Quelles sont les stratégies mises en œuvre par les écrivains pour rendre compte de la mixité linguistique d'une société de référence donnée? $\mathrm{Si}$ le domaine francophone s'est avéré particulièrement riche comme domaine d'investigation dans cette optique, c'est qu'on y trouve partout une rencontre de langues et de cultures. De l'Afrique au Québec, en passant par les Antilles, le français coexistent avec d'autres langues, et le frottement linguistique et culturel qui en résulte produit des effets intéressants dans le domaine de la littérature. Pour rendre compte de l'attitude active de l'écrivain qui se trouve ainsi, dans son entre-deux-langues, dans la nécessité d'opter pour une stratégie spécifique, Lise Gauvin a proposé le terme heureux de surconscience linguistique, terme qu'elle a déjà présenté et discuté dans plusieurs articles, et qu'elle reprend ici dans son «Introduction» en se référant entre autres à Bakhtine et à Derrida. 